\title{
A consensus on the medical treatment of acromegaly
}

Andrea Giustina, Philippe Chanson, David Kleinberg, Marcello D. Bronstein, David R. Clemmons, Anne Klibanski, Aart J. van der Lely, Christian J. Strasburger, Steven W. Lamberts, Ken K. Y. Ho, Felipe F. Casanueva and Shlomo Melmed

\begin{abstract}
In March 2013, the Acromegaly Consensus Group met to revise and update guidelines for the medical treatment of acromegaly. The meeting comprised experts skilled in the medical management of acromegaly. The group considered treatment goals covering biochemical, clinical and tumour volume outcomes, and the place in guidelines of somatostatin receptor ligands, growth hormone receptor antagonists and dopamine agonists, and alternative modalities for treatment including combination therapy and novel treatments. This document represents the conclusions of the workshop consensus.
\end{abstract}

Giustina, A. et al. Nat. Rev. Endocrinol. 10, 243-248 (2014); published online 25 February 2014; doi:10.1038/nrendo.2014.21

\section{Introduction}

In 2005, the Acromegaly Consensus Group developed a consensus statement on the medical management of acromegaly. ${ }^{1}$ Acromegaly is usually the result of a growth hormone $(\mathrm{GH})$-secreting pituitary adenoma, ${ }^{2}$ leading to anatomical changes and metabolic dysfunction caused by elevated GH and insulin-like growth factor I (IGF-I) levels. However, disease activity might persist even after surgery to remove the adenoma. Accordingly, management of patients with acromegaly is problematic, complex and costly, and requires approaches tailored to each individual patient. ${ }^{3}$ In March 2013, consensus guidelines on medical treatment of acromegaly were updated and revised at a meeting involving over 50 experts who have extensive experience in acromegaly management.

Multimodal treatment is often required to control acromegaly by suppressing $\mathrm{GH}$ hypersecretion, reducing IGF-I levels, and controlling tumour growth, leading to symptom control and minimizing the associated clinical signs and comorbidities. Surgical, pharmacological and radiotherapeutic approaches are used to treat acromegaly, and consensus statements and guidelines on acromegaly management and management of complications have been updated. ${ }^{4-7}$ Moreover, during the 2013 meeting, detailed guidance on pharmacological options for acromegaly treatment was revisited by the group and current recommendations are presented here. Recommendations were graded on the basis of the Grading of Recommendations Assessment, Development and Evaluation system (Box 1) ${ }^{8,9}$

\section{Competing interests}

S.W.L. declares no competing interests. All other authors declare competing interests. See the article online for full details of the relationships.

\section{Treatment goals \\ Biochemical outcomes}

Elevated GH and IGF-I levels are predictors of mortality in patients with acromegaly (HQ), ${ }^{10}$ and lowering GH and normalizing IGF-I levels in patients with acromegaly results in mortality rates similar to those expected in the general population (MQ). ${ }^{11}$ However, the definition of a safe GH level (in terms of normalizing mortality rates) is likely to be outdated because the data were collected retrospectively using less sensitive assays than those in routine use nowadays. Using sensitive and specific assays the cut-off for GH levels is likely to be $<1 \mu \mathrm{g} / \mathrm{l}$ (MQ).

GH and IGF-I assays of greater specificity and sensitivity are currently being standardized and validated. The numerical treatment targets using these newer assays require reassessing in the clinical setting. A 2011 consensus paper providing guidance on current GH and IGF-I assays stressed the importance of familiarity with appropriate hormone standards, specificity and sensitivity of the assay, and the need to determine assay-specific and method-specific normal GH cut-offs (SR). ${ }^{12}$

We recommend that the aim of medical treatment is to reduce fasting morning GH and IGF-I concentrations to levels that are as close to normal as possible, following the recommendations of the previous consensus meeting held in 2009 (SR), ${ }^{13}$ which were based on the existing epidemiological data. These studies should be updated using modern, sensitive and specific assays. Results of GH suppression during the oral glucose tolerance test are not useful in follow-up of medically treated patients due to inconsistent results (LQ). ${ }^{14} \mathrm{GH}$ pulsatility can be accounted for by measurement of integrated GH secretion over $24 \mathrm{~h}$, but this approach is not cost-effective. The clinical significance of slightly elevated IGF-I levels, or biochemically discordant results (between GH and IGF-I), remains to be established (VLQ). 
Box 1 | Grading of evidence and recommendations

Evidence classified as:

- Very low quality (VLQ): expert opinion with one or a small number of small uncontrolled studies in support

- Low quality (LQ): large series of small uncontrolled studies

- Moderate quality (MQ): one or a small number of large uncontrolled studies or meta-analyses

- High quality (HQ): controlled studies or large series of large uncontrolled studies with sufficiently long follow-up

Recommendations classified as:

- Discretionary recommendations (DR) if based on VLQ or LQ evidence

- Strong recommendations (SR) if based on MQ or HQ evidence

\section{Tumour shrinkage}

Medical treatment of tumours in the pituitary should prevent continued tumour growth or provide relief of symptoms and signs due to compressive mass effect, if present (SR). Markers that define tumour shrinkage (that is the percentage reduction in tumour volume) have been arbitrarily defined and are not effective, and the significance of percentage volume decrease is most likely determined by tumour location, invasiveness, size and compressive symptoms (MQ). ${ }^{15-17}$ Tumour shrinkage can occur within 3 months of starting medical treatment (LQ). ${ }^{15}$

\section{Clinical outcomes}

Clinical outcomes have been inconsistently evaluated in clinical trials of medical treatment of acromegaly; end points need to be standardized and incorporated into future prospective clinical trials (SR). Core clinical outcomes that future studies evaluating medical therapy should include as a basic dataset are: mortality; tumour volume; important comorbidities (such as hypertension and heart disease, diabetes mellitus, sleep apnoea, and bone and joint involvement); and relevant clinical symptoms such as acral changes, headache and sweating (DR). The response of comorbidities to medical treatment and/ or their reversibility need to be individually assessed in a consistent manner (SR). ${ }^{18-23}$

\section{Biochemical results with drug treatment}

Three forms of medical therapy have been used in the treatment of acromegaly: two are receptor-based, directed at the pituitary adenoma (the somatostatin receptor ligands [SRLs] octreotide and lanreotide, and the dopamine agonist cabergoline); and one is directed at decreasing and/or blocking GH effects in the periphery (the GH receptor antagonist [GHRA] pegvisomant).

\section{Somatostatin receptor ligands}

Rigorous biochemical normalization ${ }^{13}$ can be achieved by treating with SRLs in approximately $25 \%$ of unselected treatment-naive patients with acromegaly who were not previously shown to be responsive to an SRL. This figure is lower than those reported in previous guidelines and published papers that might have had patient selection bias due to the stringent inclusion and exclusion criteria required for clinical trials (MQ) ${ }^{6,18,24}$ Long-term (>3 years) results on the efficacy and safety profile of SRLs are reassuring (HQ), ${ }^{18,25,26}$ and lowering the SRL dose or decreasing the frequency of administration of SRLs might be considered for patients with long-term control of acromegaly (VLQ). ${ }^{27}$ When reducing the SRL dose or decreasing the frequency of administration, patients should be reassessed at regular intervals to ensure maintenance of therapeutic effect (SR).

Long-acting lanreotide and octreotide formulations target primarily the somatostatin receptor subtype 2 and have similar efficacy (MQ). ${ }^{18,28}$ However, lanreotide and octreotide differ in their mode of administration (long-acting lanreotide formulations are available in ready-to-use prefilled syringes that are injected subcutaneously; long-acting octreotide formulations require reconstitution before being injected intramuscularly), which might influence patient convenience (VLQ). ${ }^{24}$ At least two other SRL formulations are currently undergoing clinical development for acromegaly: pasireotide, which has a different somatostatin receptor-binding profile $^{29}$ and oral octreotide. ${ }^{30}$

\section{Dopamine agonist}

The best response to high-dose cabergoline therapy occurs in patients with mildly elevated GH levels and IGF-I levels $<2$ times the upper limit of normal (ULN), ${ }^{31}$ and if pre-treatment IGF-I levels are $>2.5 \times$ ULN, the likelihood of subsequent IGF-I normalization is low (MQ). Long-term results on the safety profile of dopamine agonists, particularly in terms of not causing cardiac valve damage, are reassuring (MQ). ${ }^{31,32}$

\section{GH receptor antagonist}

The efficacy of the GHRA pegvisomant in normalizing IGF-I levels in acromegaly is well established (HQ). ${ }^{33}$ At the appropriate dose, pegvisomant normalizes IGF-I levels in most patients (MQ). Long-term data on the efficacy and safety profile of pegvisomant are reassuring and few long-term serious adverse events have been reported (MQ), ${ }^{33}$ but ongoing vigilance is required to monitor liver function and tumour size (SR). ${ }^{33}$ Lipodystrophy can occur at the injection site (LQ). ${ }^{34}$

A discrepancy exists between the proportion of patients achieving normal IGF-I levels during treatment with pegvisomant in randomized controlled trials and community-based databases, suggesting differences in participant selection, dose titration, patient compliance, history of earlier irradiation, and occurrence of adverse events (LQ). ${ }^{33,35-37}$ If long-term control of acromegaly is achieved with pegvisomant, down-titration of the dose or decreasing the frequency of administration might be possible (DR).

\section{Clinical outcomes with drug treatment Mortality}

Mortality rates are decreased with medical treatment of acromegaly and the consequent normalization of GH and IGF-I levels (MQ), but the relative effect of different 


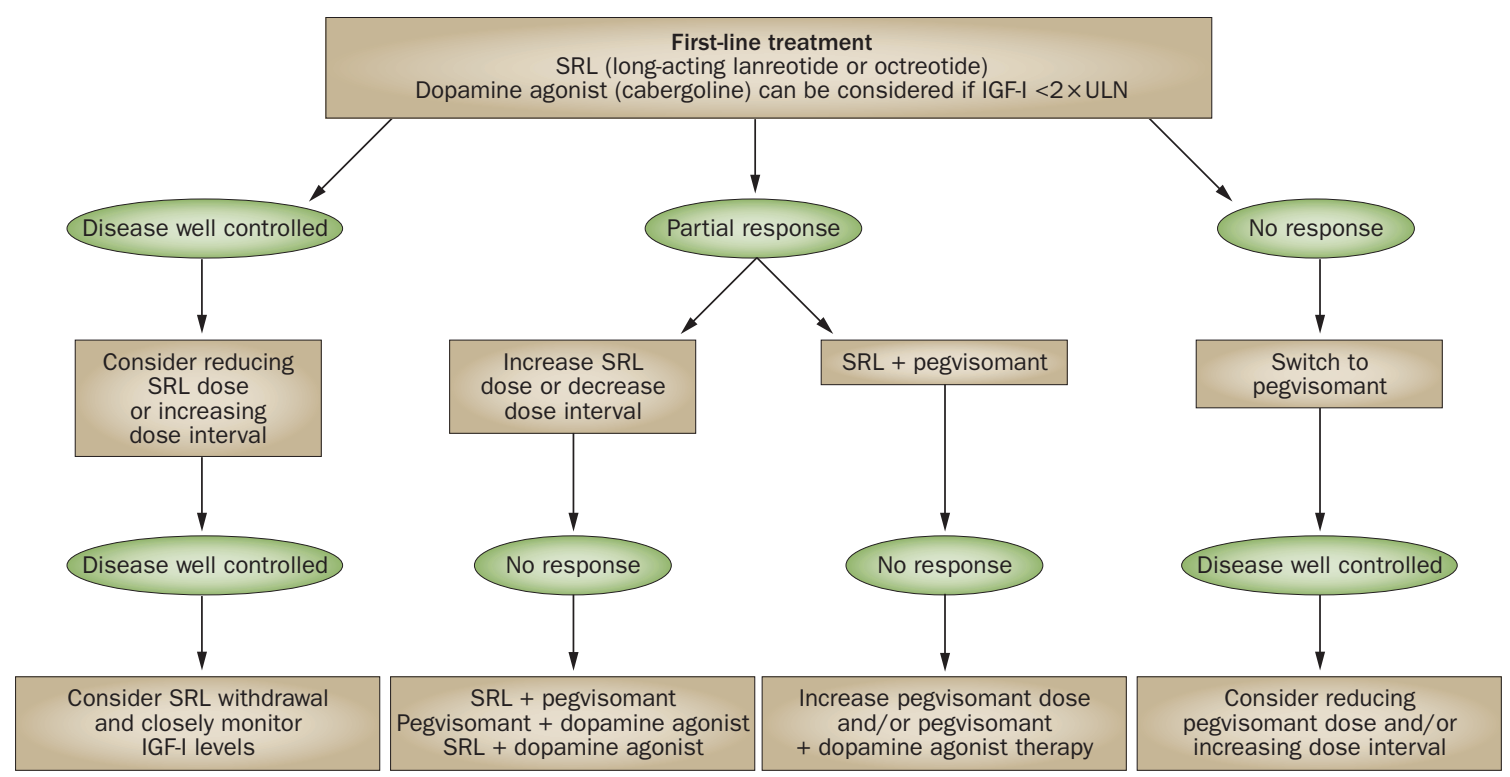

Figure 1 | Medical management of patients with acromegaly. A proposed algorithm for the medical management of acromegaly after surgery or as primary treatment strategy when surgery is inappropriate. Radiation therapy as rescue therapy has not been considered in this algorithm as its use is usually determined by a multidisciplinary management team. Abbreviations: IGF-I, insulin-like growth factor I; SRL, somatostatin receptor ligand; ULN, upper limit of normal.

specific treatments on longevity is not known (VLQ). ${ }^{11,38,39}$ Caution is needed regarding the use of conventional radiation therapy owing to reports of increased mortality and morbidity, as well as development of local tissue damage (DR), but more data are needed on mortality and treatment-induced comorbidities with currently used focused techniques such as stereotactic radiation techniques.

\section{Comorbidities}

Comorbidities related to acromegaly should be managed as they are in the general population because they lead to increased mortality (SR). Pharmacological treatment of acromegaly improves left ventricular hypertrophy and dysfunction (HQ), hypertension (LQ), and obstructive sleep apnoea (MQ). ${ }^{40-44}$ However, pharmacological treatment of acromegaly might not improve arthropathy (LQ), and effects on soft-tissue tumours are unknown. New techniques are needed to measure bone and joint integrity to assess better the effects of acromegaly treatment (MQ). ${ }^{45-48}$ Different medications for acromegaly have different effects on glucose metabolism. SRLs might have a negative influence on glucose metabolism, but generally with marginal clinical relevance and most frequently in patients who do not have biochemical control of the disease. ${ }^{6}$ By contrast, pegvisomant usually has beneficial effects on glucose metabolism. ${ }^{6}$ Blood glucose and $\mathrm{HbA}_{1 \mathrm{c}}$ levels should be monitored in all patients and managed accordingly (SR). ${ }^{49}$

\section{Socioeconomic impact of acromegaly}

The benefits to patients and their quality of life are the key consideration in medical management of acromegaly. Although the cost-effectiveness of different treatments is an important consideration in management decisions in acromegaly, ${ }^{20}$ compelling data on the cost-effectiveness of different medical options is lacking. The expertise of the pituitary surgeon influences cost-effectiveness by optimizing surgical outcomes (MQ), and the potential of radiation therapy as a means of controlling drug expenditure could also be considered (DR).$^{50-52}$

\section{Tumour volume and medical therapy}

Tumour shrinkage is commonly observed with SRL therapy concordant with a reduction in GH secretion (MQ) ${ }^{15-17}$ and normally occurs within 3 months of initiating therapy with SRLs (LQ) and continues thereafter (MQ). ${ }^{53}$ However, tumour re-growth might be observed after SRL therapy is discontinued (LQ).${ }^{54}$ Efficacy data of dopamine agonists on tumour shrinkage are sparse. ${ }^{31}$ When clinically significant tumour shrinkage has been reported, the tumours are usually mixed $\mathrm{GH}$ /prolactin secreting tumours (VLQ). ${ }^{31}$ Current data show that pegvisomant therapy rarely leads to $\mathrm{GH}$-producing pituitary tumour growth (MQ). ${ }^{33,55-57}$

\section{Recommendation for medical therapy Primary treatment}

Surgery is the primary treatment option when an experienced surgeon is available and the tumour is resectable, especially for small well-circumscribed adenomas (SR). SRLs are the primary medical treatment option if surgery is not appropriate (for example, in patients with medical contraindications such as recent myocardial infarction; if surgery is delayed; or when the patient refuses the surgical option) (SR). ${ }^{42,58-60}$ For macroadenomas, pre-surgical SRL treatment might improve outcomes, but prospective data are limited with regards to the benefit or harm of this treatment option (LQ). ${ }^{61}$ When assessing postsurgical hormone-related outcomes in patients receiving pre-surgical SRL treatment, the drug carry-over effect 
Box 2 | Key recommendations

- When considering the biochemical goals of medical treatment, a familiarity with appropriate hormone standards, assay specificity and assay sensitivity, and the determination of assay-specific and method-specific normal GH cut-offs are strongly recommended

- The importance of tumour volume decrease with medical treatment is most likely to be determined by tumour location, invasiveness, size and presence of compressive symptoms

- Long-term results are now available on the efficacy and safety profile of all medical treatments, but response rates in unselected populations of patients with acromegaly might be lower than those reported in published reports, perhaps owing to patient selection bias

- More-specific recommendations are provided for first-line and second-line post-surgical medical treatment, up-titration and down-titration of doses, switching medical therapy, and combination medical therapies (Figure 1)

should also be considered (that is, the impact of presurgical SRL treatment on subsequent post-surgical GH and IGF-I levels) (SR) and should initially be re-measured 3 and 6 months post-operatively.

\section{First-line treatment post-surgery}

SRLs are the primary first-line therapy after surgery (SR) (Figure 1). No evidence has been reported of a difference in the efficacy of long-acting lanreotide and octreotide formulations (MQ). ${ }^{16,17,28}$ Primary therapy with cabergoline might be considered in patients with mild disease (IGF-I <2 $\times$ ULN) (DR). ${ }^{6}$ Cabergoline therapy might be assessed with a short-term (3-6 months) trial with dose escalation from 1.5 to $3.5 \mathrm{mg}$ per week if well tolerated (DR). ${ }^{31}$

\section{Second-line and alternative treatments}

Patients who do not respond to SRL therapy (those in whom GH and IGF-I levels undergo minimal change) should be switched to pegvisomant treatment (SR). ${ }^{33,62,63}$ In patients who do not respond (biochemically) to medical monotherapy, combination therapy with SRL and cabergoline or pegvisomant and cabergoline can be considered, on the basis of individual clinical considerations including tumour size and location (DR). ${ }^{6,64}$ In patients having undergone radiation therapy, medical therapy might be required until effects are evident (SR).

In patients who partially respond to SRL therapy (in terms of reduction in GH and IGF-I levels or tumour shrinkage), combination therapy with pegvisomant and SRL should be considered (DR). ${ }^{6,64}$ In patients demonstrating clear decreases in GH/IGF-I levels (but in whom these levels are not normalized) when treated with the highest approved SRL doses, further dose increases or a decrease in injection interval can be considered (DR). ${ }^{65-67}$

In patients with well-controlled acromegaly during SRL therapy, a decrease in SRL administration to the minimally effective dose can be considered (DR). If both biochemical and clinical disease control are maintained with the minimal dose of SRL, an increased dose interval (up to every 3 months) can be considered (DR). If IGF-I levels remain normal with this regimen, drug withdrawal may be considered in rare cases of persistent optimum control despite progressive dose reduction (DR), but lifelong monitoring of IGF-I levels should be maintained in these patients (SR). ${ }^{68-71}$

\section{Novel agents}

New pharmacological approaches to the treatment of acromegaly in advanced stages of clinical development include new SRLs with different somatostatin receptor binding profiles (such as pasireotide) ${ }^{72}$ and oral octreotide, which uses a transient permeability enhancer to enable gut absorption. ${ }^{30}$ Novel therapeutic approaches in early stages of clinical development include an antisense oligonucleotide of 20 bases that binds to the $\mathrm{GH}$ receptor mRNA and inhibits translation of the receptor protein, and a targeted secretion inhibitor, comprising a botulinum toxin-GH-releasing hormone $(\mathrm{GHRH})$ chimera molecule that binds to cells expressing GHRH receptors, internalizes botulinum toxin and inhibits $\mathrm{GH}$ secretion. ${ }^{73}$ Temozolomide, an alkylating agent that induces DNA damage thereby effecting tumour cell death, has been assessed for $\mathrm{GH}$-aggressive pituitary tumours resistant to conventional therapy. ${ }^{74,75}$ Further study results are required to assess the potential role of these agents in the medical therapy of acromegaly.

\section{Conclusions}

Our key recommendations for the management of acromegaly are summarized in Figure 1 and Box 2. Optimal use of monotherapy or combination therapy can achieve biochemical remission in most patients with acromegaly, with durable efficacy and long-term maintained safety profiles. Assuming good treatment adherence from patients, loss of efficacy over time with medical therapy is rarely encountered. GH deficiency is not frequent but is a risk if patients are over-treated. However, patient selection bias in many reports of medical treatment outcomes for acromegaly reinforces the need for prospective studies.

Review criteria
Meeting participants were assigned to specific topics
related to acromegaly treatment and conducted literature
searches using PubMed for English language papers,
published between January 2005 and March 2013.
Search terms included "acromegaly" and terms
associated with each topic: "pathology", "medical
treatments", "biochemical goals", "tumour shrinkage",
"clinical outcomes", "dopamine agonists", "GH receptor
antagonist", "somatostatin receptor ligands", "mortality",
"comorbidities", "socioeconomic impact", "pre-surgical
treatment", "combination treatments", and "guidelines".
Assigned participants presented on these topics during
the meeting, participants divided into three subgroups for
discussion of each topic and reported to the main group.
All participants developed consensus recommendations
based on all reports presented.


1. Melmed, S. et al. Consensus statement: medica management of acromegaly. Eur. J. Endocrinol. 153, 737-740 (2005).

2. Melmed, S. Acromegaly pathogenesis and treatment. J. Clin. Invest. 119, 3189-3202 (2009).

3. Giustina, A. et al. Current management practices for acromegaly: an international survey. Pituitary 14, 125-133 (2011).

4. Melmed, S. et al. A consensus on the diagnosis and treatment of acromegaly complications. Pituitary 16, 294-302 (2013).

5. Melmed, S. et al. Guidelines for acromegaly management: an update. J. Clin. Endocrinol. Metab. 94, 1509-1517 (2009).

6. Katznelson, L. et al. American Association of Clinical Endocrinologists medical guidelines for clinical practice for the diagnosis and treatment of acromegaly-2011 update: executive summary. Endocr. Pract. 17, 636-646 (2011).

7. Melmed, S. Medical progress: acromegaly. N. Engl. J. Med. 355, 2558-2573 (2006).

8. Funder, J. W. et al. Case detection, diagnosis, and treatment of patients with primary aldosteronism: an Endocrine Society clinical practice guideline. J. Clin. Endocrinol. Metab. 93, 3266-3281 (2008).

9. Guyatt, G. H. et al. GRADE: an emerging consensus on rating quality of evidence and strength of recommendations. BMJ 336 , 924-926 (2008).

10. Holdaway, I. M., Rajasoorya, R. C. \& Gamble, G. D. Factors influencing mortality in acromegaly. J. Clin. Endocrinol. Metab. 89, 667-674 (2004).

11. Holdaway, I. M., Bolland, M. J. \& Gamble, G. D. A meta-analysis of the effect of lowering serum levels of GH and IGF-I on mortality in acromegaly. Eur. J. Endocrinol. 159, 89-95 (2008)

12. Clemmons, D. R. Consensus statement on the standardization and evaluation of growth hormone and insulin-like growth factor assays. Clin. Chem. 57, 555-559 (2011).

13. Giustina, A. et al. A consensus on criteria for cure of acromegaly. J. Clin. Endocrinol. Metab. 95, 3141-3148 (2010).

14. Carmichael, J. D., Bonert, V. S., Mirocha, J. M. $\&$ Melmed, S. The utility of oral glucose tolerance testing for diagnosis and assessment of treatment outcomes in 166 patients with acromegaly. J. Clin. Endocrinol. Metab. 94, 523-527 (2009).

15. Melmed, S. et al. A critical analysis of pituitary tumor shrinkage during primary medical therapy in acromegaly. J. Clin. Endocrinol. Metab. 90 4405-4410 (2005).

16. Mazziotti, G. \& Giustina, A. Effects of lanreotide SR and Autogel on tumor mass in patients with acromegaly: a systematic review. Pituitary 13 60-67 (2010).

17. Giustina, A. et al. Meta-analysis on the effects of octreotide on tumor mass in acromegaly. PLOS ONE 7, e36411 (2012).

18. Colao, A., Auriemma, R. S., Galdiero, M., Lombardi, G. \& Pivonello, R. Effects of initial therapy for five years with somatostatin analogs for acromegaly on growth hormone and insulinlike growth factor-l levels, tumor shrinkage, and cardiovascular disease: a prospective study. J. Clin. Endocrinol. Metab. 94, 3746-3756 (2009).

19. Colao, A. et al. Octreotide LAR vs. surgery in newly diagnosed patients with acromegaly: a randomized, open-label, multicentre study. Clin. Endocrinol. (Oxf.) 70, 757-768 (2009).

20. Ben-Shlomo, A., Sheppard, M. C., Stephens, J. M., Pulgar, S. \& Melmed, S. Clinical, quality of life, and economic value of acromegaly disease control. Pituitary 14, 284-294 (2011).
21. Davi, M. V. et al. Sleep apnoea syndrome is highly prevalent in acromegaly and only partially reversible after biochemical control of the disease. Eur. J. Endocrinol. 159, 533-540 (2008).

22. Espinosa-de-los-Monteros, A. L., Gonzalez, B., Vargas, G., Sosa, E. \& Mercado, M. Clinical and biochemical characteristics of acromegalic patients with different abnormalities in glucose metabolism. Pituitary 14, 231-235 (2011)

23. Bonadonna, S. et al. Increased prevalence of radiological spinal deformities in active acromegaly: a cross-sectional study in postmenopausal women. J. Bone Miner. Res. 20, 1837-1844 (2005)

24. Salvatori, R. et al. Effectiveness of self- or partner-administration of an extended-release aqueous-gel formulation of lanreotide in lanreotide-naive patients with acromegaly. Pituitary 13, 115-122 (2010).

25. Yang, L. P. \& Keating, G. M. Octreotide long acting release (LAR): a review of its use in the management of acromegaly. Drugs 70 , 1745-1769 (2010)

26. Toledano, Y. et al. Efficacy of long-term lanreotide treatment in patients with acromegaly. Pituitary 12, 285-293 (2009).

27. Schopohl, J. et al. Efficacy and acceptability of lanreotide Autoge ${ }^{\circledR} 120 \mathrm{mg}$ at different dose intervals in patients with acromegaly previously treated with octreotide LAR. Exp. Clin. Endocrinol. Diabetes 119, 156-162 (2011).

28. Murray, R. D. \& Melmed, S. A critical analysis of clinically available somatostatin analog formulations for therapy of acromegaly. J. Clin. Endocrinol. Metab. 93, 2957-2968 (2008).

29. Petersenn, S. et al. Pasireotide (SOM230) demonstrates efficacy and safety in patients with acromegaly: a randomized, multicenter, phase II trial. J. Clin. Endocrinol. Metab. 95, 2781-2789 (2010).

30. Tuvia, S. et al. Oral octreotide absorption in human subjects: comparable pharmacokinetics to parenteral octreotide and effective growth hormone suppression. J. Clin. Endocrinol. Metab. 97, 2362-2369 (2012).

31. Sandret, L., Maison, P. \& Chanson, P. Place of cabergoline in acromegaly: a meta-analysis. J. Clin. Endocrinol. Metab. 96, 1327-1335 (2011).

32. Maione, L. et al. No evidence of a detrimenta effect of cabergoline therapy on cardiac valves in patients with acromegaly. J. Clin. Endocrinol. Metab. 97, E1714-E1719 (2012).

33. van der Lely, A. J. et al. Long-term safety of pegvisomant in patients with acromegaly: comprehensive review of 1288 subjects in ACROSTUDY. J. Clin. Endocrinol. Metab. 97, 1589-1597 (2012).

34. Bonert, V. S. et al. Lipodystrophy in patients with acromegaly receiving pegvisomant. J. Clin. Endocrinol. Metab. 93, 3515-3518 (2008).

35. Garcia Basavilbaso, N. et al. Experience from the Argentine Pegvisomant Observational Study: preliminary data. Front. Horm. Res. 38, 42-49 (2010).

36. Sievers, C. et al. Change of symptoms and perceived health in acromegalic patients on pegvisomant therapy: a retrospective cohort study within the German Pegvisomant Observational Study (GPOS). Clin. Endocrinol. (Oxf.) 73, 89-94 (2010).

37. Trainer, P. J., Ezzat, S., D'Souza, G. A., Layton, G. \& Strasburger, C. J. A randomized, controlled, multicentre trial comparing pegvisomant alone with combination therapy of pegvisomant and long-acting octreotide in patients with acromegaly. Clin. Endocrinol. (Oxf.) 71, 549-557 (2009).
38. Dekkers, O. M., Biermasz, N. R., Pereira, A. M., Romijn, J. A. \& Vandenbroucke, J. P. Mortality in acromegaly: a metaanalysis. J. Clin. Endocrinol. Metab. 93, 61-67 (2008).

39. Burgers, A. M. et al. Meta-analysis and doseresponse metaregression: circulating insulin-like growth factor I (IGF-I) and mortality. J. Clin. Endocrinol. Metab. 96, 2912-2920 (2011).

40. Colao, A. et al. No greater incidence or worsening of cardiac valve regurgitation with somatostatin analog treatment of acromegaly. J. Clin. Endocrinol. Metab. 93, 2243-2248 (2008).

41. Gouya, H. et al. Rapidly reversible myocardial edema in patients with acromegaly: assessment with ultrafast T2 mapping in a single-breath-hold MRI sequence. AJR Am. J. Roentgenol. 190, 1576-1582 (2008).

42. Annamalai, A. K. et al. A comprehensive study of clinical, biochemical, radiological, vascular, cardiac, and sleep parameters in an unselected cohort of patients with acromegaly undergoing presurgical somatostatin receptor ligand therapy. J. Clin. Endocrinol. Metab. 98, 1040-1050 (2013).

43. Attal, P. \& Chanson, P. Endocrine aspects of obstructive sleep apnea. J. Clin. Endocrinol. Metab. 95, 483-495 (2010).

44. Berg, C. et al. Influence of disease control with pegvisomant on sleep apnoea and tongue volume in patients with active acromegaly. Eur. J. Endocrinol. 161, 829-835 (2009).

45. Biermasz, N. R. et al. Automated image analysis of hand radiographs reveals widened joint spaces in patients with long-term control of acromegaly: relation to disease activity and symptoms. Eur. J. Endocrinol. 166, 407-413 (2012)

46. Claessen, K. M. et al. Increased clinical symptoms of acromegalic arthropathy in patients with long-term disease control: a prospective follow-up study. Pituitary 17 44-52 (2014).

47. Mazziotti, G. et al. Prevalence of vertebral fractures in men with acromegaly. J. Clin. Endocrinol. Metab. 93, 4649-4655 (2008).

48. Battista, C. et al. Spinal volumetric trabecular bone mass in acromegalic patients: a longitudinal study. Clin. Endocrinol. (Oxf.) 70, 378-382 (2009)

49. Mazziotti, G. et al. Effects of somatostatin analogs on glucose homeostasis: a metaanalysis of acromegaly studies. J. Clin. Endocrinol. Metab. 94, 1500-1508 (2009).

50. Roset, M. et al. Cost of clinical management of acromegaly in Spain. Clin. Drug Investig. 32 235-245 (2012)

51. Sesmilo, G. et al. Changes in acromegaly treatment over four decades in Spain: analysis of the Spanish Acromegaly Registry (REA). Pituitary 16, 115-121 (2013).

52. Bex, M. et al. AcroBel—the Belgian registry on acromegaly: a survey of the 'real-life' outcome in 418 acromegalic subjects. Eur. J. Endocrinol. 157, 399-409 (2007).

53. Bevan, J. S. Clinical review: the antitumoral effects of somatostatin analog therapy in acromegaly. J. Clin. Endocrinol. Metab. 90, 1856-1863 (2005).

54. van der Lely, A. J. et al. Control of tumor size and disease activity during cotreatment with octreotide and the growth hormone receptor antagonist pegvisomant in an acromegalic patient. J. Clin. Endocrinol. Metab. 86, 478-481 (2001)

55. Jimenez, C. et al. Follow-up of pituitary tumor volume in patients with acromegaly treated with pegvisomant in clinical trials. Eur. J. Endocrinol. 159, 517-523 (2008). 


\section{REVIEWS}

56. Buhk, J. H. et al. Tumor volume of growth hormonesecreting pituitary adenomas during treatment with pegvisomant: a prospective multicenter study. J. Clin. Endocrinol. Metab. 95, 552-558 (2010).

57. Marazuela, M. et al. Somatotroph tumor progression during pegvisomant therapy: a clinical and molecular study. J. Clin. Endocrinol. Metab. 96, E251-E259 (2011).

58. Carlsen, S. M. et al. Six-month preoperative octreotide treatment in unselected, de novo patients with acromegaly: effect on biochemistry, tumour volume, and postoperative cure. Clin. Endocrinol. (Oxf.) 74, 736-743 (2011).

59. Mao, Z. G. et al. Preoperative lanreotide treatment in acromegalic patients with macroadenomas increases short-term postoperative cure rates: a prospective, randomised trial. Eur. J. Endocrinol. 162, 661-666 (2010).

60. Shen, M. et al. Effect of presurgical long-acting octreotide treatment in acromegaly patients with invasive pituitary macroadenomas: a prospective randomized study. Endocr. J. 57, 1035-1044 (2010).

61. Pita-Gutierrez, F. et al. Place of preoperative treatment of acromegaly with somatostatin analog on surgical outcome: a systematic review and meta-analysis. PLoS ONE 8, e61523 (2013).

62. Trainer, P. J. ACROSTUDY: the first 5 years. Eur. J. Endocrinol. 161 (Suppl. 1), S19-S24 (2009).

63. Higham, C. E., Chung, T. T., Lawrance, J., Drake, W. M. \& Trainer, P. J. Long-term experience of pegvisomant therapy as a treatment for acromegaly. Clin. Endocrinol. (Oxf.) 71, 86-91 (2009).

64. Neggers, S. J. \& van der Lely, A. J. Combination treatment with somatostatin analogues and pegvisomant in acromegaly. Growth Horm. IGF Res. 21, 129-133 (2011).

65. Giustina, A. et al. High-dose intramuscular octreotide in patients with acromegaly inadequately controlled on conventional somatostatin analogue therapy: a randomised controlled trial. Eur. J. Endocrinol. 161, 331-338 (2009).
66. Mazziotti, G. et al. Effects of high-dose octreotide LAR on glucose metabolism in patients with acromegaly inadequately controlled by conventional somatostatin analog therapy. Eur. J. Endocrinol. 164, 341-347 (2011).

67. Fleseriu, M. Clinical efficacy and safety results for dose escalation of somatostatin receptor ligands in patients with acromegaly: a literature review. Pituitary 14, 184-193 (2011).

68. Auriemma, R. S. et al. Complete disappearance of a GH-secreting pituitary macroadenoma in a patient with acromegaly: effect of treatment with lanreotide Autogel and consequence of treatment withdrawal. Eur. J. Endocrinol. 162, 993-999 (2010).

69. Ozbek, M., Erdogan, M., Akbal, E. \& Gonulalan, G. Disappearance of a GH secreting macroadenoma, during long-term somatostatin analogue administration. Exp. Clin. Endocrinol. Diabetes 117, 309-311 (2009).

70. Ramirez, C. et al. Discontinuation of octreotide LAR after long term, successful treatment of patients with acromegaly: is it worth trying? Eur. J. Endocrinol. 166, 21-26 (2012).

71. Ronchi, C. L. et al. Preliminary data on biochemical remission of acromegaly after somatostatin analogs withdrawal. Eur. J. Endocrinol. 158, 19-25 (2008).

72. Colao, A. et al. Pasireotide LAR is significantly more effective than octreotide LAR at inducing biochemical control in patients with acromegaly: results of a 12-month randomized, double-blind, multicenter, phase III study. Endocrine Abstr. 29, OC1.1 (2012)

73. Somm, E. et al. A botulinum toxin-derived targeted secretion inhibitor downregulates the GH/IGF1 axis. J. Clin. Invest. 122, 3295-3306 (2012).

74. Morin, E., Berthelet, F., Weisnagel, J., Bidlingmaier, M. \& Serri, O. Failure of temozolomide and conventional doses of pegvisomant to attain biochemical control in a severe case of acromegaly. Pituitary 15, 97-100 (2012).
75. Hagen, C., Schroeder, H. D., Hansen, S., Hagen, C. \& Andersen, M. Temozolomide treatment of a pituitary carcinoma and two pituitary macroadenomas resistant to conventional therapy. Eur. J. Endocrinol. 161 631-637 (2009).

\section{Acknowledgements}

The authors thank all participants in the Ninth Acromegaly Consensus Group meeting: Michel Aubert (Switzerland), John Ayuk (UK), Ariel Barkan (USA), Albert Beckers (Belgium), Paolo Beck-Peccoz (Italy), Anat Ben Shlomo (USA), Nienke Biermasz (Netherlands), Jens Bollerslev (Norway), Philippe Caron (France), Kazuo Chihara (Japan), Annamaria Colao (Italy), Ettore Degli Uberti (Italy), Eleni Dimarki (USA), William Drake (UK), Diego Ferone (Italy), Maria Fleseriu (USA), Hidenori Fukuoka (Japan), Monica Gadelha (Brazil), Yona Greenman (Israel), Ashley Grossman (UK), Peter Kamenicky (France), Larry Katznelson (USA), Marta Korbonits (UK), Andre Lacroix (Canada), Anton Luger (Austria), Gherardo Mazziotti (Italy), Moises Mercado (Mexico), Sebastian Neggers (Netherlands), Vera Popovic-Brkic (Serbia), Johannes Romijn (Netherlands), Jochen Schopohl (Germany), Gunther Stalla (Germany), Peter Trainer (UK), Jacqueline Trouillas (France), Stylianos Tsagarakis (Greece), Mary Lee Vance (USA), John Wass (UK), Susan Webb (Spain), Margaret Wierman (USA) and Whitney Woodmansee (USA). This study was sponsored by an unrestricted educational grant from Ipsen Ltd, Paris, France. Scientific sponsorship of the meeting was provided by Cedars-Sinai Medical Center. We also acknowledge the editorial assistance provided by Martin Gilmour (ESP Bioscience, Crowthorne, UK), who attended the workshop to take minutes that were used to assist the authors in writing.

Author contributions

S.M. and A.G. wrote the majority of the manuscript. All authors made substantial contribution to discussion of content and reviewed/edited the manuscript before submission. 